\title{
Grain Orientation Induced Softening in Electrodeposited Gradient Nanostructured Nickel during Cold Rolling Deformation
}

https://doi.org/10.1515/rams-2020-0105

Received Aug 10, 2019; accepted Dec 17, 2019

\begin{abstract}
Quantitative microstructural evolution and the corresponding microhardness of electrodeposited nanostructured nickel sheet during cold rolling deformation are investigated by $\mathrm{x}$-ray diffraction, transmission electron microscopy and Vicker's microhardness testing. Particularly, to investigate the effect of stress states on deformation behavior, two series of gradient nanostructured nickel with symmetric structures and the homogeneous counterparts with three levels of grain size are compared based on macro-statistical data. In such hierarchical sandwich-like gradient samples, the layers with larger grain size, as the soft phase, indeed sustain more deformation. Deformation-induced grain rotation changes are observed in the center layers with a relatively larger grain size, accompanied by an obvious decrease in microhardness. According to the quantitative microstructural parameters including the grain size, dislocation density and stacking fault probability before and after deformation, evaluation based on Hall-Petch and Bailey-Hirsch relationships indicates the transition from strain hardening to softening can be attributed to grain orientation change.
\end{abstract}

Keywords: gradient structured metal, nickel, cold rolling, microstructure, microhardness

\footnotetext{
*Corresponding Author: Jiang Zhu: Chongqing Key Laboratory of Environmental Materials \& Remediation Technologies, Chongqing University of Arts and Sciences, Chongqing, 402160, China; Email: Jiangzhu415@163.com

Haitao Ni, Zhaodong Wang: College of Chemistry and Environmental Engineering, Chongqing University of Arts and Sciences, Chongqing, 402160, China

Lixia Wang: College of Intelligent Manufacturing Engineering, Chongqing University of Arts and Sciences, Chongqing, 402160, China
}

๖ Open Access. ๑ 2020 H. Ni et al., published by De Gruyter. (cc) BY License

\section{Introduction}

The strengthening-toughening technology of nanostructured metals has always been one of the most active research fields in mechanics and materials science [1-3]. Due to the inverted relationship between the strength and toughness of metallic materials, high strength nanostructured metals with homogeneous microstructure often exhibit poor plasticity, which seriously restricts their practical application in engineering. Recently, the concept of microstructural gradient has been increasingly applied to engineering materials [4-10]. Relevant research results demonstrate that gradient nanostructured materials can possess both high strength and good plasticity. Further analysis reveals that the establishment of gradient structure can distribute stress better, and the plasticity of nanostructured metal can be improved remarkably by the coordination of strain caused by the inconsistency of plastic deformation $[3,4,7]$. Nevertheless, with the increasing demands of industrial development on the comprehensive properties of metals, how to thoroughly understand the effect of structural gradient on mechanical properties is a key problem when developing high-performance gradient metallic materials.

There can be no doubt that the characterization of the microstructures and the strain (stress) gradient effects of gradient nanostructured metals is a basic scientific problem that urgently needs to be solved through experimental investigation. Taking gradient rate as an example, even within the same range of grain size change, different gradient distributions (gradient rates) will lead to different microstructural evolution and mechanical property change $[11,12]$. Compared to their homogeneous counterparts, the discrepancy in the deformation mechanism of gradient nanostructured metals is still not fully clear. Therefore, in the present paper, to better grasp the relationship between the microstructure and properties of gradient nanostructures, the evolutions of microstructures and microhardness of typical layers (regions) with different grain sizes during rolling deformation deserve to be dis- 
cussed quantitatively. In view of the fact that the average grain size for the majority of homogeneous samples is concentrated in the range of $20-100 \mathrm{~nm}$ in the previous studies on electrodeposited nanostructured nickel, for a more comprehensive comparison of some relevant information on the evolution of the microstructure and performance, the grain size gradient-structured sample is designed with a narrow grain size distribution ranging from $20 \mathrm{~nm}$ to $100 \mathrm{~nm}$. Furthermore, in order to understand the effect of stress state on the microstructure and the properties of gradient microstructures, a comparison between homogeneous and gradient samples is also made.

\section{Materials and Methods}

As shown in Figure 1, two series of gradient nanostructured nickel with symmetric structures were selected as the research materials. For simplicity, the gradient sample with high surface hardness but low core hardness was referred to as sample $H L H$, and the gradient sample with low surface hardness but high core hardness was referred to as sample $L H L$. The detailed preparation process and the microstructure of the electrodeposited gradient structure can be found in a previously reported investigation [13], except for electrodeposition time. Considering surface grinding of test samples for microstructure and hardness analysis, the electrodeposition time of surface layer in the sample $L H L$ was extended to double duration. Furthermore, several homogeneous samples with typical grain sizes, prepared by the same electrodeposition device, were used for comparative analysis.

The as-prepared sheets were cut into small pieces with same dimensions: $6 \mathrm{~mm} \times 5 \mathrm{~mm}$. Several pieces of samples with similar initial microstructures, selected by Xray diffraction (XRD) analysis, were sent through a pair of rollers with a diameter of $180 \mathrm{~mm}$ at room temperature. The nominal rolling strain of each sample was determined by $\epsilon=2 \ln \left(t_{0} / t\right) / \sqrt{3}$, where $t_{0}$ and $t$ are the initial thickness and final thickness, respectively. To have a thorough knowledge of the microstructural evolution of the deformed gradient samples, for each deformed sample, five locations were selected as information collection points. According to our previous investigation, the average grain size in these layers were observed to be $\sim 22 \mathrm{~nm}, \sim 45 \mathrm{~nm}$, and $\sim 90 \mathrm{~nm}$, respectively. For simplicity, these layers with different sizes were named layer A, B, and C. On the basis of electrodeposition rate, it can be calculated that layer $\mathrm{B}$ is located at $1 / 4$ thickness from the surface of sample $H L H$ and $1 / 8$ thickness from the surface of sample $L H L$.
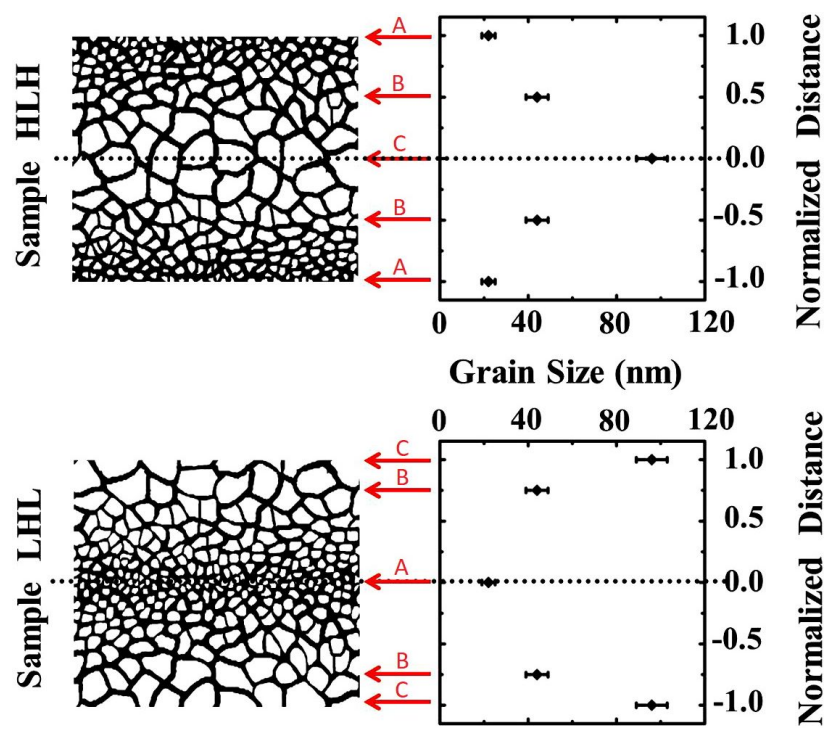

Figure 1: Schematic diagram of two types of gradient nanostructured nickel with symmetrical structure.

The deformation-induced microstructural changes were quantitatively examined by XRD analysis using D/MAX-2500 PC diffractometer with a rotary Cu target (18 $\mathrm{KW}$ ), which was operated in the fixed-time scan mode $\left(0.02^{\circ}\right.$ per step) from $40^{\circ}$ to $100^{\circ}$ with a step time of 0.5 s. Semi-quantitative microstructural information concerning grain size and defect density was obtained from X-ray line profile analysis. To verify the results obtained from XRD analysis, transmission electron microscopy (TEM) was employed to intuitively evaluate the final microstructure of the ND-RD sections, particularly the grain size distribution. Additionally, considering the limited dimensions of the samples, microhardness measurements were conducted on all deformed samples by HVS-1000A microhardness tester using a load of $0.98 \mathrm{~N}$ and a dwell time of 10 seconds.

\section{Results and discussions}

Figure 2 shows the microhardness results for the homogeneous and gradient nanostructured nickel samples with various rolling strains. For the homogeneous nanostructured samples, it can be seen that the microhardness of the typical layers increases gradually and monotonously with increasing nominal rolling strain. In addition, for layers at different locations, there is no obvious difference at the same or similar rolling strain. These results agree well with previous studies within the range of such small deformations [14, 15]. However, for the gradient nanostructured 

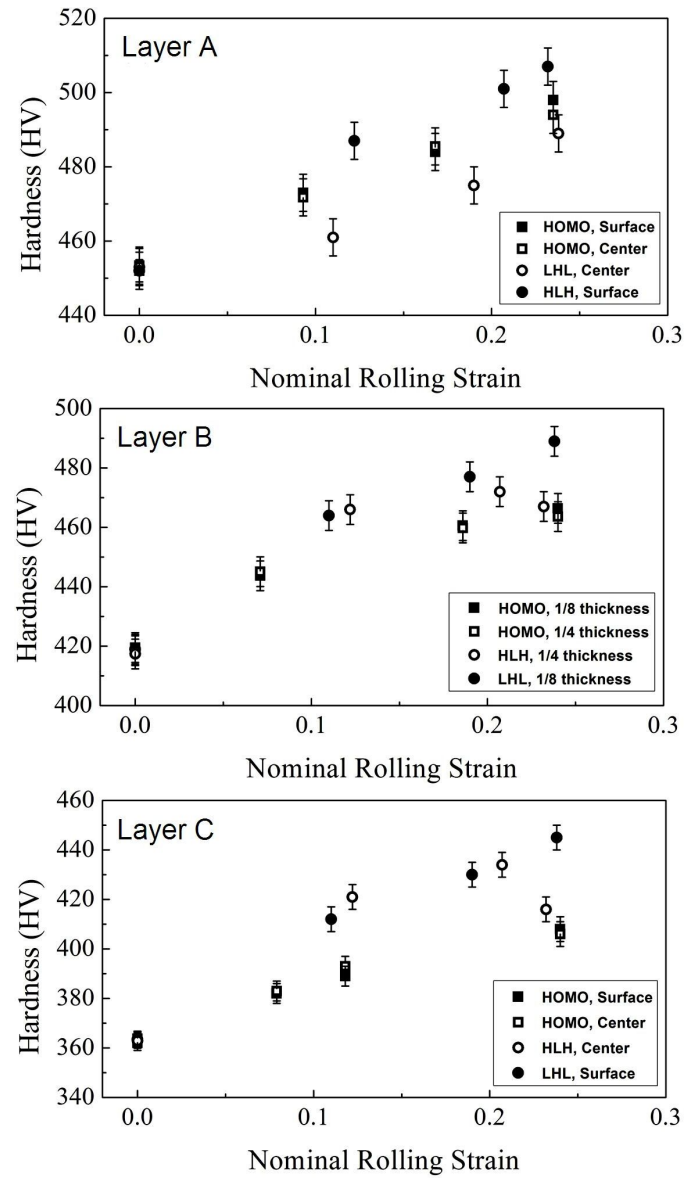

Figure 2: Microhardness evolution of typical layers in the gradient and homogeneous nanostructured nickel samples at different rolling strains.

samples, the microhardness evolution of the typical layers with different grain sizes is somewhat different.

Taking layer $\mathrm{C}$ as an example, the hardness at the surface location of sample $L H L$ exhibits an obvious increase of $\sim 80 \mathrm{HV}$ with increasing rolling strain. This increase is much larger than the increase of the deformed homogenous sample. Such a difference may be related to the strengthening mechanism of the gradient microstructure $[2,16,17]$. On the basis of the reported deformation mechanism [2], the large grains, as the "soft" phase in the sample structure, will be deformed first. Compared to a homogenous structure, they should sustain more deformation during the whole rolling process. More crystal defects will be generated and accumulated. In contrast, the "hard" phase with small grains will sustain less deformation, and the increase in hardness of the "hard" phase will be lower than that of the "soft" phase under the same deformation rate. To better understand the evolution discrepancy of microhardness for the gradient samples, microhardness evolution of two series of gradient samples are redrawn and
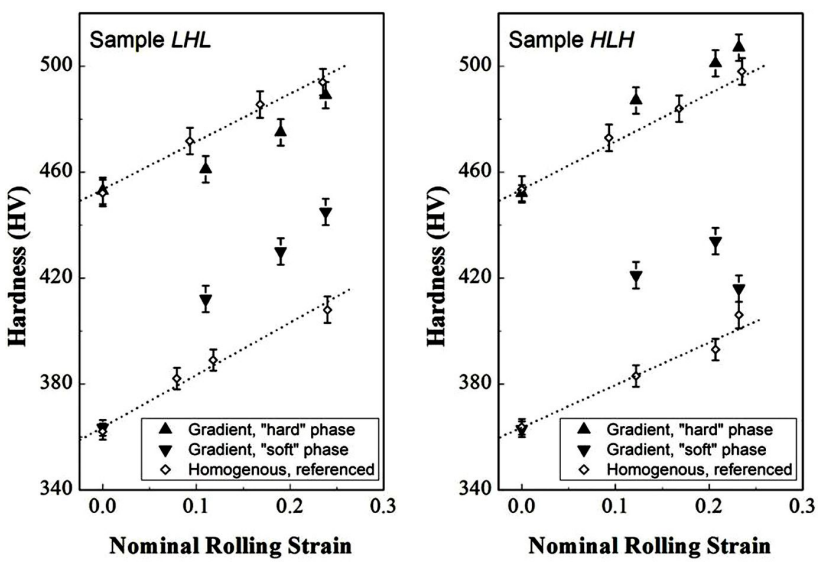

Figure 3: Microhardness evolution of gradient samples relative to their corresponding homogeneous counterparts.
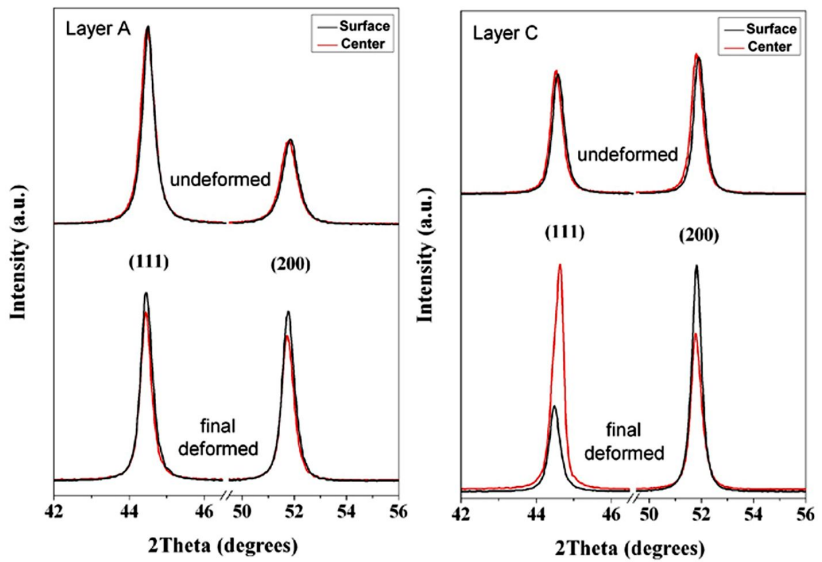

Figure 4: Typical XRD patterns for the layer $A$ and layer $C$ in the different positions of undeformed and final-deformed gradient samples.

illustrated in Figure 3. It can be clear seen that the microhardness variation of the soft phase is bigger than that of the hard phase, except the final value of the surface hard phase and the center soft phase for the sample $H L H$, which is almost same. At the center location (soft phase) of sample $H L H$, the microhardness of layer $\mathrm{C}$ first increases and then decreases, reaching a final value of $\sim 416 \mathrm{HV}$, which is still higher than the value of the deformed homogenous sample at the maximum strain. A similar transition phenomenon has also been found in layer B at 1/4 thickness from the surface of sample $H L H$. This transition should be related to the evolution of the deformation microstructures.

Figure 4 shows the typical XRD patterns for the layer $\mathrm{A}$ and layer $\mathrm{C}$ in the undeformed and final-deformed gradient samples. From the visual comparison on the widths and intensities of (111) and (200) peaks, it can be clearly seen that there are some distinct differences in microstruc- 

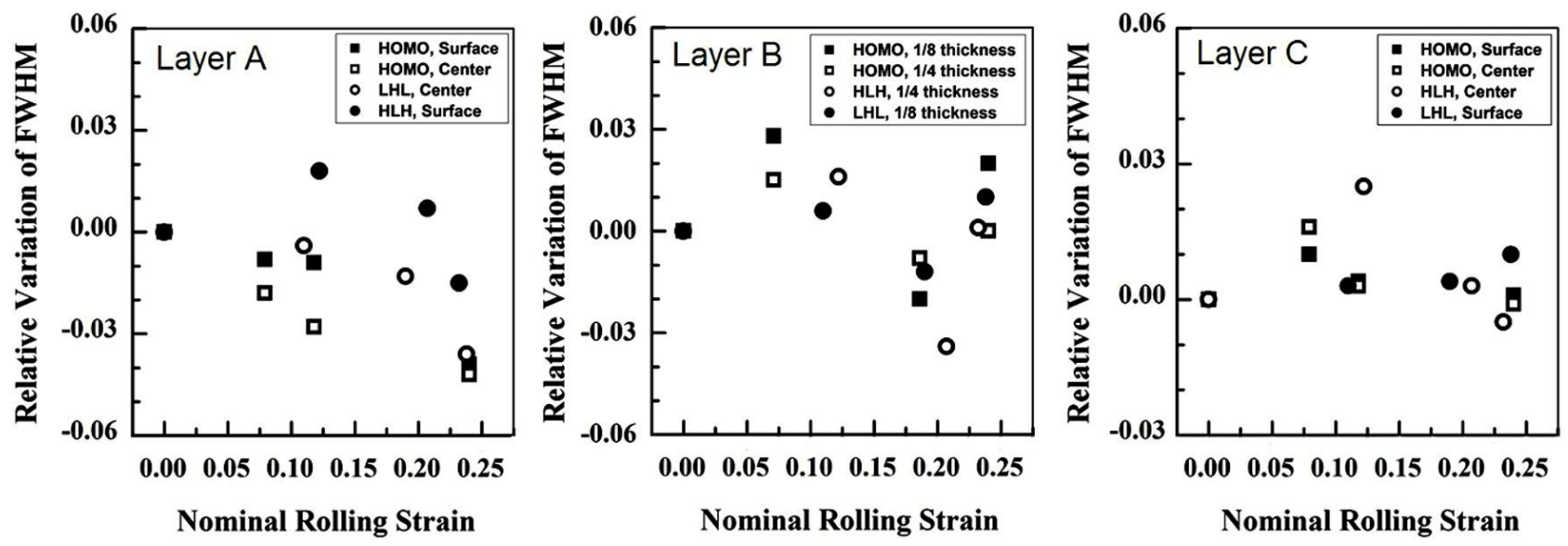

Figure 5: The relative variation of FWHM of the (111) peak versus nominal rolling strain.

Table 1: Grain size (d), dislocation density $(\rho)$ and stacking fault probability $(\alpha)$ determined by $x$-ray line profile analysis.

\begin{tabular}{|c|c|c|c|c|c|c|c|}
\hline \multirow[t]{2}{*}{ Sample } & \multirow[t]{2}{*}{ Layer } & \multicolumn{3}{|c|}{ Undeformed } & \multicolumn{3}{|c|}{ Final deformed } \\
\hline & & $\begin{array}{c}d \\
(n m)\end{array}$ & $\begin{array}{c}\rho \\
\left(\times 10^{15} / \mathrm{m}^{2}\right)\end{array}$ & $\begin{array}{c}\alpha \\
\left(\times 10^{-3}\right)\end{array}$ & $\begin{array}{c}d \\
(n m)\end{array}$ & $\begin{array}{c}\rho \\
\left(\times 10^{15} / \mathrm{m}^{2}\right)\end{array}$ & $\begin{array}{c}\alpha \\
\left(\times 10^{-3}\right)\end{array}$ \\
\hline$L H L$ & C & 76 & 3.0 & 4.0 & 95 & 3.8 & 2.6 \\
\hline$L H L$ & B & 40 & 2.9 & 5.3 & 64 & 3.5 & 4.3 \\
\hline$L H L$ & A & 22 & 2.6 & 2.2 & 30 & 2.9 & 5.2 \\
\hline$H L H$ & A & 22 & 2.7 & 2.0 & 31 & 3.7 & 4.9 \\
\hline$H L H$ & B & 40 & 3.0 & 5.8 & 59 & 3.9 & 5.4 \\
\hline$H L H$ & C & 76 & 3.0 & 4.0 & 93 & 3.4 & 5.6 \\
\hline
\end{tabular}

tural evolution characteristics between deformed layers with different average grain sizes. Furthermore, the layer with similar grain size but different positions exhibit different evolution paths, under two kinds of stress state. To further understand the differences in microstructure, a series of XRD patterns are quantitatively analyzed.

Figure 5 shows the change in full widths at half maximum (FWHM) for the homogeneous and gradient samples with various nominal rolling strains. Variation in FWHM is a well-known parameter used to investigate microstructural changes $[18,19]$. In particular, grain size, dislocation density and stacking fault probability for the undeformed and final deformed layers for the gradient samples are determined and summarized in Table 1. Based on our previous investigation of the microstructure of cold-rolled nanostructured nickel [20, 21], FWHM variation is mainly influenced by grain size and dislocation density. With increasing rolling strain, an increase in the FWHM of the (111) diffraction peak is found in the majority of the deformed layers. Considering that stress-induced grain growth behavior is commonly observed in deformed nanostructured metals, even in the gradient microstructure [22, 23], grain size should not increase FWHM values, and thus an increase in FWHM indicates dislocation accumulation in the deformed grains. It has also been observed that FWHM decreases in some deformed layers, especially in layers with small grain size. The main reason for this decrease could be attributed to the negative effect of grain growth on peak broadening. Under stress, tiny grains are easily grown due to grain rotation and the coalescence mechanism [24-27]. Relative to grains with medium and large sizes, grain rotation activity is significantly more pronounced, which is confirmed by the change of $I_{111} / I_{200}$, as shown in Figure 6 . This size dependence of grain rotation agrees well with other studies on nanostructured materials $[24,26]$.

Figure 6 shows the ratio of the (111) and (200) diffraction intensities $I_{111} / I_{200}$ for the homogeneous and gradient samples with various nominal rolling strains. The ratio of peak diffraction intensities is commonly used to indirectly evaluate grain rotation activity. Deformation induced grain rotation is found in both homogeneous and gradient samples. Furthermore, the effect of two different stress states on deformation behavior is also revealed by the change rate of $I_{111} / I_{200}$. On the whole, since the 


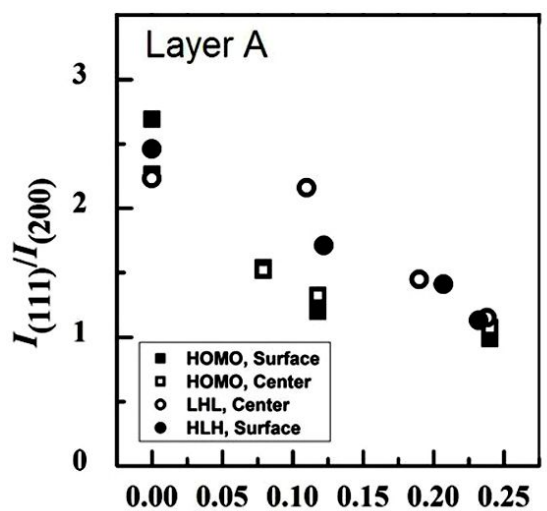

Nominal Rolling Strain

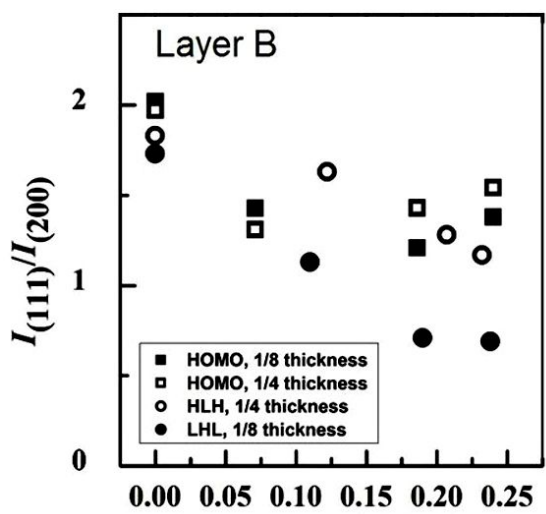

Nominal Rolling Strain

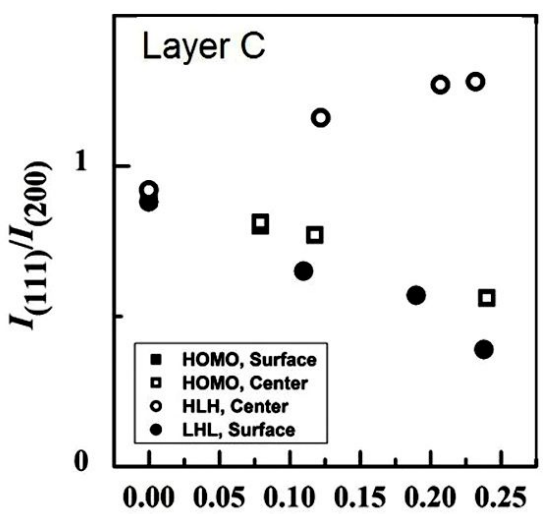

Nominal Rolling Strain

Figure 6: The ratio of the (111) and (200) diffraction intensities $I_{111} / I_{200}$ versus nominal rolling strain.
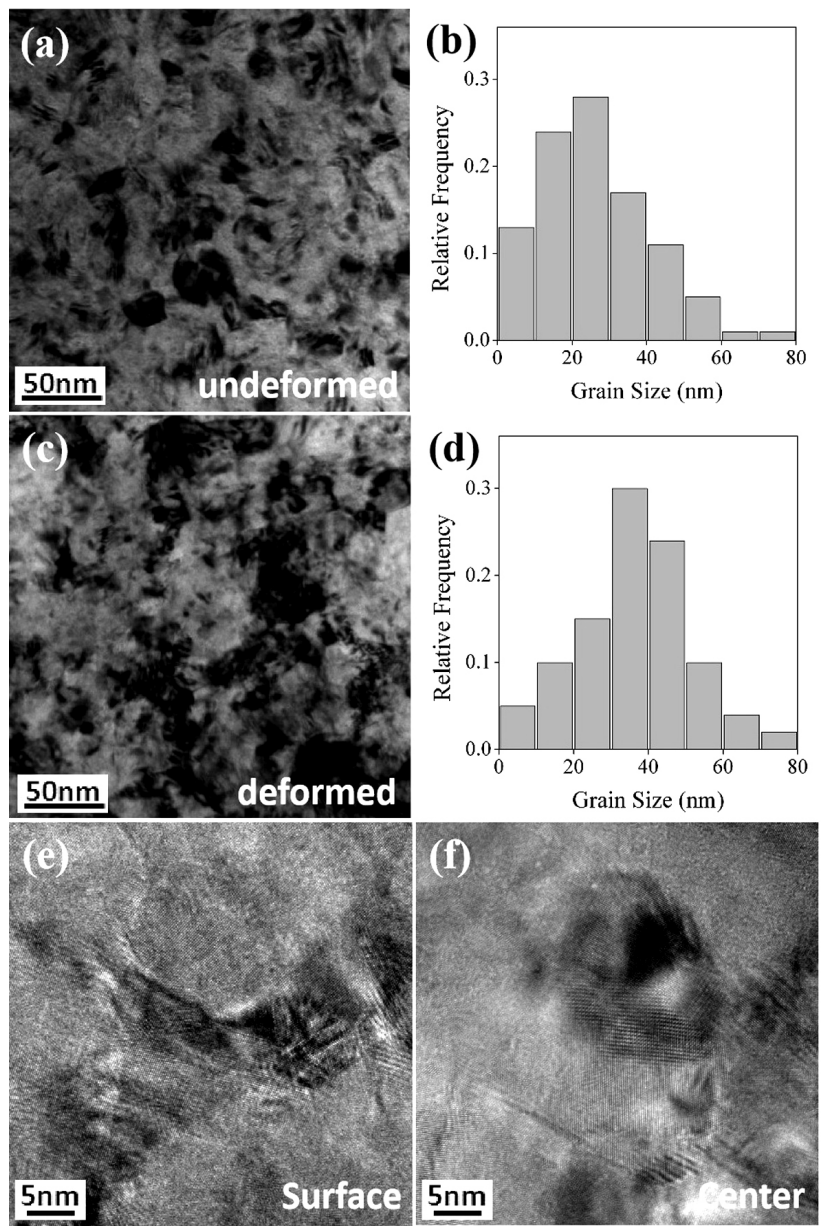

Figure 7: Typical TEM results of layer A in the gradient nanostructured samples: bright-field TEM images of (a) undeformed and (c) final-deformed samples; corresponding grain size distributions of (b) undeformed and (d) final-deformed samples; HRTEM images of (e) surface layer in sample $H L H$ and (f) center layer in sample $L H L$ after final deformation. surface layers simultaneously undergo compression and shear deformation $[21,28]$, the surface or near surface layers exhibit a relatively higher variation ratio of $I_{111} / I_{200}$. For the center layer, it would be closer to deformation in plane strain compression [21, 29], the preferential (200) orientation is relatively weak. In the case of the sample $H L H$, the center layer even exhibits reversal change of $I_{111} / I_{200}$.

Figure 7 shows the typical TEM results of layer A in gradient nanostructured samples. Overall, the observed microstructural information, such as grain size and defect density, supports the XRD analysis. Statistical results on the grain size distribution indicate that there is indeed evident grain coarsening. Furthermore, the dislocation density in the surface layer of sample $H L H$ is significantly higher than that in the center layer of sample $L H L$, as seen from typical high-resolution TEM images.

On the basis of quantitative microstructural parameters and microhardness, more attention should be paid to the relationship between the microstructural evolution and the mechanical behavior of gradient nanostructured samples, particularly the transition from hardening to softening. According to the classic Hall-Petch relation and Bailey-Hirsch relation, the combined effects of grain size and dislocation density on microhardness evolution can typically be well observed. During the rolling process, despite the grains continuing to grow, strain hardening behavior still occurs due to increasing dislocation density. Nevertheless, there seem to be some exceptions. Taking layer $\mathrm{B}$ as an example, after the final deformation, layer B at $1 / 4$ thickness from the surface of sample $H L H$ has a smaller grain size and a higher dislocation density compared to layer $B$ at $1 / 8$ thickness from the surface of sample $L H L$, but the hardness of the former is slightly lower than that of the latter. Similar differences also appear in layer C. It is difficult to explain the microhardness gap 
based on the combined effects of grain size and dislocation density without considering the possible interaction between the crystal defects. From the stacking fault probability values, the final-deformed layer $\mathrm{C}$ at the center of sample $H L H$ should have more stack faults, indirectly implying a stronger interaction between crystal defects. Further analysis indicates that deformation-induced grain orientation change could account for the decreased microhardness value. From the evolution of $I_{111} / I_{200}$, there are obvious differences between the surface layer and the center layer. Several experiments on the Hall-Petch relationship have reported grain orientation or texture effects on the Hall-Petch slope [30,31]. It can be generally concluded that the stronger the texture is, the larger the slope. Moreover, quantitative comparison of different textures shows that $k_{100}$ is greater than $k_{110}$. In the hierarchical sandwichlike structure of gradient samples, as seen in the center or near-center layers, the preferential (100) orientation weakens with increasing rolling strain. Instead, the preferential (110) orientation becomes stronger under the compressive stress component. Therefore, when the grain size and dislocation density are approximately equal, the center or near-center layers with preferential (110) orientation will be softer than the surface or near-surface layers with preferential (100) orientation.

\section{Conclusions}

In summary, the microstructural evolution and microhardness of two types of electrodeposited gradient nanostructured nickel samples with symmetric structure during coldrolling deformation are quantitatively investigated. Particularly, the effects of two stress states on the evolution characteristics of deformed layers with different average sizes are compared. Under similar deformation conditions, there are some discrepancies in the microstructural evolution as well as the mechanical behavior between gradient and homogeneous samples. Based on the analysis of all currently available data, grain orientation change induced softening is found in the gradient samples. It is expected that these results will provide a theoretical basis and technical support for the structure design, processing, and application of gradient nanostructured metals.

Acknowledgement: The authors gratefully acknowledge the National Natural Science Foundation of China (grant number 51601026) and Local Fund for Chongqing University of Arts and Sciences (grant number R2018SJD19).
Conflict of Interests: The authors declare no conflict of interest.

\section{References}

[1] Ma, E., and T. Zhu. Towards strength-ductility synergy through the design of heterogeneous nanostructures in metals. Materials Today, Vol. 20, No. 6, 2017, pp. 323-331.

[2] Lu, K. Nanomaterials. Making strong nanomaterials ductile with gradients. Science, Vol. 345, No. 6203, 2014, pp. 1455-1456.

[3] Wu, X. L., P. Jiang, L. Chen, J. F. Zhang, F. P. Yuan, and Y. T. Zhu. Synergetic Strengthening by Gradient Structure. Materials Research Letters, Vol. 2, No. 4, 2014, pp. 185-191.

[4] Moering, J., X. Ma, J. Malkin, M. Yang, Y. Zhu, and S. Mathaudhu. Synergetic strengthening far beyond rule of mixtures in gradient structured aluminum rod. Scripta Materialia, Vol. 122, 2016, pp. 106-109.

[5] Zhu, L., H. Ruan, A. Chen, X. Guo, and J. Lu. Microstructures-based constitutive analysis for mechanical properties of gradientnanostructured 304 stainless steels. Acta Materialia, Vol. 128, 2017, pp. 375-390.

[6] Wang, P. F., Z. Han, and K. Lu. Enhanced tribological performance of a gradient nanostructured interstitial-free steel. Wear, Vol. 402-403, 2018, pp. 100-108.

[7] Zeng, Z., X. Li, D. Xu, L. Lu, and T. Zhu. Gradient plasticity in gradient nano-grained metals. Extreme Mechanics Letters, Vol. 8, 2015, pp. 213-219.

[8] Ding, J., Q. Li, J. Li, S. Xue, Z. Fan, H. Wang, and X. Zhang. Mechanical behavior of structurally gradient nickel alloy. Acta Materialia, Vol. 149, 2018, pp. 57-67.

[9] Deng, S. Q., A. Godfrey, W. Liu, and N. Hansen. A gradient nanostructure generated in pure copper by platen friction sliding deformation. Scripta Materialia, Vol. 117, 2016, pp. 41-45.

[10] Yin, Z., L. Sun, J. Yang, Y. L. Gong, and X. K. Zhu. Mechanical behavior and deformation kinetics of gradient structured $\mathrm{Cu}-\mathrm{Al}$ alloys with varying stacking fault energy. Journal of Alloys and Compounds, Vol. 687, 2016, pp. 152-160.

[11] Lin, Y., J. Pan, H. F. Zhou, H. J. Gao, and Y. Li. Mechanical properties and optimal grain size distribution profile of gradient grained nickel. Acta Materialia, Vol. 153, 2018, pp. 279-289.

[12] Chen, Z. and Y. Chen Y. Nanocrystalline gradient engineering: Grain evolution and grain boundary networks. Computational Materials Science, Vol. 141, 2018, pp. 282-292.

[13] Ni, H. T., P. Li, Z. D. Wang, Z. X. Zou, M. C. Zhao, L. X. Wang, and J. Zhu. Fabrication and Characterization of Nanocrystalline Nickel with a Grain Size Gradient by Direct Current Electrodeposition. International Journal of Electrochemical Science, Vol. 14, 2019, pp. 8429-8438.

[14] Zhang, X. Y., Q. Liu, X. L. Wu, and A. W. Zhu. Work softening and annealing hardening of deformed nanocrystalline nickel. Applied Physics Letters, Vol. 93, No. 26, 2008, pp. 261907.

[15] Cao, Z. H., P. Y. Li, L. Wang, Z. H. Jiang, and X. K. Meng. A crossover from hardening to softening in nanocrystalline $\mathrm{Ni}$ by annealing and rolling. Applied Physics. A, Materials Science \& Processing, Vol. 109, No. 3, 2012, pp. 613-619.

[16] Yang, M. X., Y. Pan, F. P. Yuan, Y. T. Zhu, and X. L. Wu. Back stress strengthening and strain hardening in gradient structure. Mate- 
rials Research Letters, Vol. 4, No. 3, 2016, pp. 145-151.

[17] Wang, Y. F., C. X. Huang, M. S. Wang, Y. S. Li, and Y. T. Zhu. Quantifying the synergetic strengthening in gradient material. Scripta Materialia, Vol. 150, 2018, pp. 22-25.

[18] Zhang, K., I. V. Alexandrov, R. Z. Valiev, and K. Lu. Structural characterization of nanocrystalline copper by means of $\mathrm{x}$-ray diffraction. Journal of Applied Physics, Vol. 80, No. 10, 1996, pp. 5617-5624.

[19] Ungár, T. E. Schafler E, and J. Gubicza. In Bulk Nanostructured Materials edited by M.J. Zehetbauer and by Y.T. Zhu, Wiley-VCH, Weinheim, 2009, pp. 361-386.

[20] Ni, H. T., and X. Y. Zhang. Effect of iron content on mechanical behavior of nanocrystalline nickel and alloys. Materials Science and Engineering A, Vol. 541, 2012, pp. 216-221.

[21] Ni, H., H. Lv, Z. Wang, J. Zhu, and X. Zhang. Comparative Study on Microstructural Stability of Pre-annealed Electrodeposited Nanocrystalline Nickel During Pack Rolling. Nanoscale Research Letters, Vol. 13, No. 1, 2018, pp. 337.

[22] Chen, W., Z. S. You, N. R. Tao, Z. H. Jin, and L. Lu. Mechanicallyinduced grain coarsening in gradient nano-grained copper. Acta Materialia, Vol. 125, 2017, pp. 255-264.

[23] Ni, H. T., J. Zhu, Z. D. Wang, H. Y. Lv, Y. Y. Su, and X. Y. Zhang. A brief overview on grain growth of bulk electrodeposited nanocrystalline nickel and nickel-iron alloys. Reviews on Advanced Materials Science, Vol. 58, No. 1, 2019, pp. 98-106.

[24] Chen, B., K. Lutker, J. Lei, J. Yan, S. Yang, and H. K. Mao. Detecting grain rotation at the nanoscale. Proceedings of the National Academy of Sciences of the United States of America, Vol. 111, No. 9, 2014, pp. 3350-3353.
[25] Li, J., A. K. Soh, and X. Wu. On nanograin rotation by dislocation climb in nanocrystalline materials. Scripta Materialia, Vol. 78-79, 2014, pp. 5-8.

[26] Moldovan, D., D. Wolf, S. R. Phillpot, and A. J. Haslam. Trends in Nanoscale Mechanics. Harik V. M. and M. D. Salas, Eds. Springer, Holland, 2003, Chapter 2, pp. 35-59.

[27] Vuppuluri, A., and S. Vedantam. Grain growth rate for coupled grain boundary migration and grain rotation in nanocrystalline materials. Philosophical Magazine Letters, Vol. 96, No. 9, 2016, pp. 339-346.

[28] Madhavan, R., S. Nagaraju, and S. Suwas. Texture Evolution in Nanocrystalline Nickel: Critical Role of Strain Path. Metallurgical and Materials Transactions. A, Physical Metallurgy and Materials Science, Vol. 46, No. 2, 2015, pp. 915-925.

[29] Lohmiller, J., M. Grewer, C. Braun, A. Kobler, C. Kübel, K. Schüler, V. Honkimäki, H. Hahn, O. Kraft, R. Birringer, and P. A. Gruber. Untangling dislocation and grain boundary mediated plasticity in nanocrystalline nickel. Acta Materialia, Vol. 65, 2014, pp. 295307.

[30] Yuan, R., I. J. Beyerlein, and C. Zhou. Coupled crystal orientationsize effects on the strength of nano crystals. Scientific Reports, Vol. 6, No. 1, 2016, id. 26254, DOI: https://doi.org/10.1038/ srep26254.

[31] Godon, A., J. Creus, S. Cohendoz, E. Conforto, X. Feaugas, P. Girault, and C. Savall. Effects of grain orientation on the HallPetch relationship in electrodeposited nickel with nanocrystalline grains. Scripta Materialia, Vol. 62, No. 6, 2010, pp. 403406. 